\title{
Avaliação estética da montagem dos seis dentes superiores anteriores em prótese total ${ }^{\dagger}$
}

\section{Esthetic evaluation of the setting up of the six anterior superior teeth in complete dentures}

\author{
Osmar Vieira de CASTRO Jr.* \\ Zlata Victorovna HVANOV** \\ Maria Luiza M. A. FRIGERIO***
}

\begin{abstract}
CASTRO Jr., O. V. de; HVANOV, Z. V.; FRIGERIO, M. L. M. A. Avaliação estética da montagem dos seis dentes superiores anteriores em prótese total. Pesq Odont Bras, v. 14, n. 2, p. 177-182, abr./jun. 2000.

A presente pesquisa teve por objetivo fazer uma avaliação estética de diferentes tipos de montagens de dentes anteriores superiores na prova em cera de uma prótese total. Sendo o assunto de natureza subjetiva, as próteses foram submetidas à apreciação de um grupo de pessoas entre estudantes, especialistas e leigos. Foram confeccionadas cinco próteses superiores ocluindo com uma única inferior, para cada um dos 10 pacientes do sexo masculino na faixa etária entre 18 e 72 anos. Os dentes anteriores de cada uma das próteses foram montados com um tipo de caracterização: para jovens (clássica), senil, com toque feminino, com toque masculino e com diastemas. As conclusões foram as seguintes: as montagens que mais agradaram foram a para jovens (clássica) com 38,57\%, seguida da senil com $32,85 \%$, sem diferenças significantes entre elas. As montagens que menos agradaram foram a com toque feminino com $32,86 \%$ de desaprovação, seguida da montagem masculina $(31,43 \%)$ e com diastema $(22,86 \%)$, sem diferenças significantes entre elas.
\end{abstract}

UNITERMOS: Estética dentária; Prótese total.

\section{INTRODUÇÃO}

A estética em prótese total é um assunto que envolve vários fatores que em conjunto proporcionam uma harmonia com o restante da face.

Os fatores sexo, personalidade e idade foram brilhantemente abordados nos trabalhos de FRUSH; FISCHER ${ }^{7,8,10}$. Quanto ao sexo, mencionaram que os contornos arredondados dos ângulos incisais produzindo efeito esférico dos incisivos centrais superiores e incisivos laterais superiores, se harmonizam com o sexo feminino, enquanto os ângulos retos produzindo efeito cubóide nesses mesmos dentes, se harmonizam com o sexo masculino. Quanto ao fator personalidade classificaram os indivíduos em tipo vigoroso (na sua maioria homens), médio (a maior parte da população), e delicado (na sua maioria mulheres). Quanto ao fator idade propuseram para indivíduos jovens uma montagem dos seis dentes anteriores superiores com desniveis entre as bordas incisais, e para indi- víduos senis, simulações de desgastes físiológicos como erosão, abrasão ou diastemas, variações nos eixos longitudinais dos dentes e simulação de retrações gengivais.

Em 1974, FREITAS et al. ${ }^{5}$ ressaltaram que para se obter uma boa estética deve-se considerar uma tomada correta da dimensão vertical, o plano oclusal protético, linha mediana, linha dos caninos e linha alta do sorriso, e com relação aos dentes, NICÁCIO ${ }^{14}$ (1976) apontou três fatores na seleção dos dentes artificiais: forma, tamanho e cor. Além da forma dos dentes, sua disposição é um fator fundamental para se construir uma prótese mais estética tornando-a mais natural ${ }^{1,16,20}$.

Já GOLDSTEIN ${ }^{11}$ (1980), ENGELMEIER ${ }^{3}$ (1996) e GOMES ${ }^{12}$ (1998) realçaram a importância da caracterização das bases de acordo com as necessidades de cada paciente. Outros fatores também tem influência na composição estética, entre eles: a importância da consulta de entrevista para co-

\footnotetext{
${ }^{\dagger}$ Resumo da Dissertação de Mestrado.

* Mestre em Prótese Dental; ** Professora Associada; *** Professora Doutora - Departamento de Prótese Dental da Faculdade de Odontologia da USP.
} 
CASTRO Jr., O. V. de; HVANOV, Z. V.; FRIGERIO, M. L. M. A. Avaliação estética da montagem dos seis dentes superiores anteriores em prótese total. Pesq Odont Bras, v. 14, n. 2, p. 177-182, abr./jun. 2000.
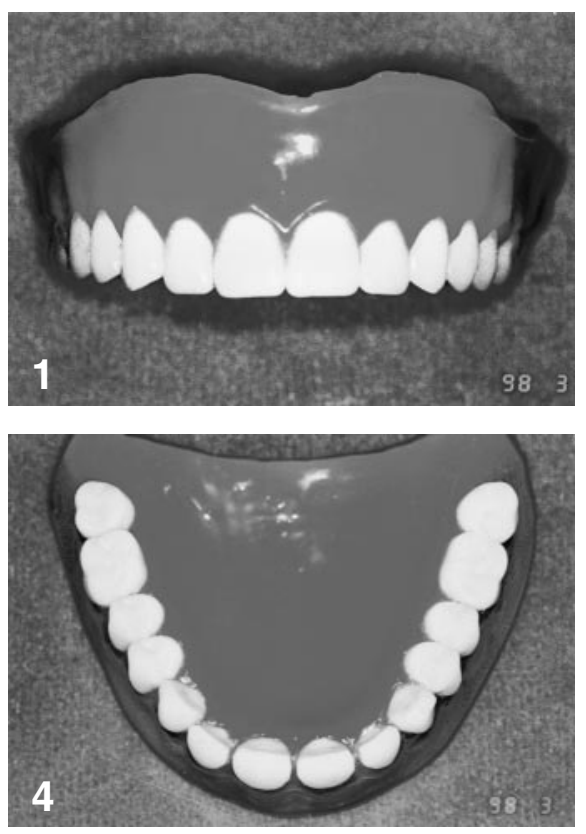
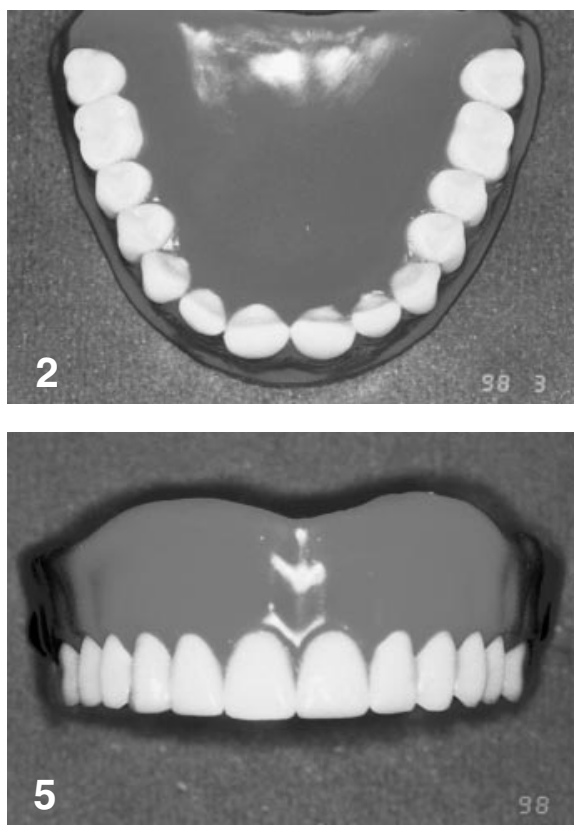

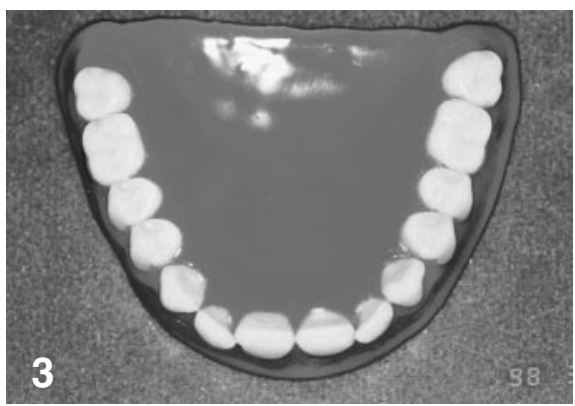

FIGURA 1 - Montagem clássica. Vista frontal.

FIGURA 2 - Montagem com toque masculino. Vista oclusal.

FIGURA 3 - Montagem com toque feminino. Vista oclusal. FIGURA 4 - Montagem com diastemas. Vista oclusal. FIGURA 5 - Montagem senil. Vista frontal. nhecer as expectativas do paciente, a tomada precisa de relação cêntrica e excêntrica, a localização do plano oclusal e a influência da própria entrega e manutenção ${ }^{2}$.

Em 1990 HEARTWELL; RAHN ${ }^{13}$ acrescentaram que fatores cosméticos e a reflexão artística devem ser considerados no arranjo dos dentes de uma prótese total.

RUFENACHT $^{15}$ (1990) citou o aspecto da morfopsicologia, isto é, como uma pessoa vê a outra. Desse ponto de vista, os incisivos centrais focalizam as características de personalidade, força, energia, autoritarismo, magnetismo, apatia ou retração. Os incisivos laterais concentram o abstrato: elementos artístico, emocional ou intelectual da personalidade. Assim, o profissional, ao confeccionar uma prótese total pode alterar totalmente a imagem do paciente, positiva ou negativamente.

Associado aos fatores para obter-se uma boa estética: tamanho dos dentes, cor, disposição, alinhamento, posição, cor da gengiva artificial, oclusão, enfim, todos os recursos utilizados para se promover um aspecto mais natural e harmônico possivel, deve-se sempre consultar a opinião do paciente que deve dar a última palavra, pois o conceito do belo é de caráter totalmente pessoal e subjetivo, sofrendo influências culturais e sociais. Procedendo desta maneira, estaremos mais próximos de realizar seu desejo estético, obtendo uma maior satisfação e aceitação do trabalho.

\section{MATERIAL E MÉTODOS}

Foram selecionados 10 pacientes desdentados completos, do sexo masculino, de cor branca, na faixa etária entre 18 e 72 anos. As fases clinicas para a confecção das próteses totais foram executadas conforme a orientação preconizada por TAMAKI; TAMAKI ${ }^{18}$ e TAMAKI ${ }^{19}$ até a fase de reprodução dos planos-de-orientação, quando eram reproduzidos cinco planos-de-orientação superior e um único inferior.

Para cada um dos pacientes selecionados foram realizadas cinco tipos de montagens diferentes dos dentes superiores que ocluíam com uma única inferior. Alterando-se a disposição ou através de desgaste dos seis dentes superiores anteriores obteve-se:

Montagem clássica: essa montagem apresenta desniveis entre as bordas dos incisivos centrais e laterais correspondendo aos arcos dentais de indivíduos na primeira fase da maturidade, na sua plenitude física, quando ainda os dentes não sofreram desgastes fisiológicos ou mecânicos (Figura 1$)$.

Montagem com toque masculino: os incisivos centrais superiores eram expostos, projetando-se o lado distal para vestibular e os incisivos laterais superiores eram deslocados de leve para palatino tornando-os menos evidentes. Com essas variações, salientavam-se os incisivos centrais superiores e os caninos e a montagem tornava-se mais agressiva (Figura 2). 
CASTRO Jr., O. V. de; HVANOV, Z. V.; FRIGERIO, M. L. M. A. Avaliação estética da montagem dos seis dentes superiores anteriores em prótese total. Pesq Odont Bras, v. 14, n. 2, p. 177-182, abr./jun. 2000.

TABELA 1 - Freqüência em números dos resultados dos votos quanto a preferência das 5 montagens (pacientes do sexo masculino).

\begin{tabular}{|c|c|c|c|c|c|c|c|c|c|c|c|c|c|}
\hline \multirow{2}{*}{$\begin{array}{c}\text { Ordem/ } \\
\text { Paciente/ } \\
\text { Idade }\end{array}$} & \multirow{2}{*}{ Montagem } & \multicolumn{5}{|c|}{ Ordem de Preferência } & \multirow{2}{*}{$\begin{array}{c}\text { Ordem/ } \\
\text { Paciente/ } \\
\text { Idade }\end{array}$} & \multirow{2}{*}{ Montagem } & \multicolumn{5}{|c|}{ OPrdem de Preferência } \\
\hline & & $1^{\mathrm{o}}$ & $2^{\mathrm{o}}$ & $3^{\circ}$ & $4^{\circ}$ & $5^{\circ}$ & & & $1^{\mathrm{o}}$ & $2^{o}$ & $3^{\circ}$ & $4^{\mathrm{o}}$ & $5^{\circ}$ \\
\hline \multirow{3}{*}{$\begin{array}{c}1 \\
\text { H.N. } \\
62 \text { anos }\end{array}$} & Diastema & 0 & 3 & 0 & 0 & 4 & \multirow{3}{*}{$\begin{array}{c}6 \\
\text { R.B. } \\
66 \text { anos }\end{array}$} & Diastema & 2 & 2 & 0 & 3 & 0 \\
\hline & Senil & 3 & 0 & 1 & 3 & 0 & & Senil & 1 & 3 & 1 & 0 & 2 \\
\hline & Feminina & 0 & 1 & 4 & 2 & 0 & & Feminina & 0 & 2 & 0 & 1 & 4 \\
\hline \multirow{5}{*}{$\begin{array}{c}2 \\
\text { P.S. } \\
72 \text { anos }\end{array}$} & Diastema & 0 & 3 & 3 & 1 & 0 & \multirow{5}{*}{$\begin{array}{c}7 \\
\text { R.G.L. } \\
62 \text { anos }\end{array}$} & Diastema & 0 & 0 & 3 & 2 & 2 \\
\hline & Clássica & 2 & 2 & 1 & 1 & 1 & & Clássica & 6 & 1 & 0 & 0 & 0 \\
\hline & Senil & 4 & 2 & 0 & 1 & 0 & & Senil & 1 & 5 & 0 & 1 & 0 \\
\hline & Feminina & 0 & 0 & 1 & 3 & 3 & & Feminina & 0 & 0 & 2 & 1 & 4 \\
\hline & Masculino & 1 & 0 & 2 & 1 & 3 & & Masculino & 0 & 1 & 2 & 3 & 1 \\
\hline \multirow{2}{*}{$\begin{array}{c}3 \\
\text { P.P. } \\
62 \text { anos }\end{array}$} & Feminina & 0 & 1 & 2 & 2 & 3 & \multirow{2}{*}{$\begin{array}{c}8 \\
\text { D.S. } \\
70 \text { anos }\end{array}$} & Feminina & 0 & 0 & 0 & 3 & 3 \\
\hline & Masculino & 1 & 2 & 2 & 3 & 0 & & Masculino & 3 & 1 & 1 & 1 & 0 \\
\hline \multirow{5}{*}{$\begin{array}{c}4 \\
\text { E.P.S. } \\
18 \text { anos }\end{array}$} & Diastema & 3 & 2 & 1 & 1 & 0 & \multirow{5}{*}{$\begin{array}{c}9 \\
\text { W.A. } \\
60 \text { anos }\end{array}$} & Diastema & 0 & 4 & 1 & 1 & 2 \\
\hline & Clássica & 0 & 3 & 1 & 0 & 3 & & Clássica & 6 & 1 & 1 & 0 & 0 \\
\hline & \begin{tabular}{|l|} 
Senil \\
\end{tabular} & 1 & 1 & 1 & 3 & 1 & & Senil & 1 & 3 & 3 & 1 & 0 \\
\hline & Feminina & 0 & 0 & 3 & 2 & 2 & & Feminina & 0 & 0 & 3 & 3 & 2 \\
\hline & Masculino & 3 & 1 & 1 & 1 & 1 & & Masculino & 1 & 0 & 0 & 3 & 4 \\
\hline \multirow{2}{*}{$\begin{array}{c}5 \\
\text { M.F.A. } \\
\text { 51 anos }\end{array}$} & Diastema & 0 & 2 & 2 & 0 & 2 & \multirow{2}{*}{$\begin{array}{c}10 \\
\text { J.R.F. } \\
68\end{array}$} & Diastema & 4 & 2 & 1 & 0 & 0 \\
\hline & Clássica & 0 & 3 & 3 & 0 & 0 & & Clássica & 0 & 5 & 1 & 1 & 0 \\
\hline
\end{tabular}

Montagem com toque feminino: a distal dos incisivos centrais superiores eram ligeiramente deslocadas para palatino e os ângulos mésio-incisais dos incisivos laterais superiores eram sobrepostos sobre a vestibular dos incisivos centrais superiores, colocando em evidência os incisivos laterais superiores (Figura 3).

Montagem com diastemas: as faces proximais dos incisivos centrais superiores e incisivos laterais superiores eram desgastadas estreitando-os. Montados os dentes nas posições originais, os mesmos ficavam separados entre si, simulando a migração daqueles ou o abrasionamento das faces proximais (Figura 4).

Montagem senil: as bordas incisais dos incisivos centrais superiores, incisivos laterais superio- res e caninos eram desgastadas, deixando-se os dentes com ângulos mais vivos e nivelados, simulando abrasão (Figura 5).

Uma vez terminadas as caracterizações das montagens, todas recebiam uma escultura convencional cuidando-se para que a altura das bordas livres da gengiva, tamanho, direção e volume das bossas, depressões dos sulcos interdentais e sulcos horizontais não diferissem entre as próteses, a fim de que não fosse incorporada nenhuma variável que pudesse interferir na avaliação.

No dia da prova clínica das próteses, reunia-se um grupo com cerca de sete pessoas. Solicitava-se que observassem atentamente a reconstituição estética da boca com cada uma das cinco próteses e logo depois manifestassem por escrito, qual a 
CASTRO Jr., O. V. de; HVANOV, Z. V.; FRIGERIO, M. L. M. A. Avaliação estética da montagem dos seis dentes superiores anteriores em prótese total. Pesq Odont Bras, v. 14, n. 2, p. 177-182, abr./jun. 2000.

montagem que julgasse mais harmoniosa ou estética ao paciente, indicando com o número 1 (um) e as demais em ordem decrescente de preferência. Assim, o número 5 seria a prótese menos apreciada.

As avaliações foram feitas individualmente, sem que a pessoa tomasse conhecimento da escolha das outras. Os avaliadores foram: o próprio paciente, estudantes de Odontologia, leigos e especialistas em Prótese Dentária.

Após computados os dados a prótese que o paciente escolhera como sendo a mais harmônica era acrilizada junto com a inferior e posteriormente instalada.

\section{RESULTADOS E DISCUSSÃO}

Após a avaliação estética dos 10 pacientes do sexo masculino, os dados foram computados e analisados quanto a freqüência em números (Tabela 1), quanto a freqüência das ordens de preferência em número, porcentagem e teste qui-quadrado (Tabela 2) e com testes qui-quadrados para opção de preferência com exclusão sucessiva dos menores valores (Tabela 3 ).

Examinando-se os resultados encontrados nesta pesquisa, apresentados nas Tabelas 1 e 2, verifica-se entre as cinco montagens dos dentes anteriores, oferecidas para apreciação, a que mais agradou aos avaliadores, em termos de reconstituição estética da região bucal, foi a do tipo clássico, com $38,57 \%$ dos votos; seguida de perto, a montagem senil em segundo lugar, com 32,85\%

TABELA 2 - Freqüência das ordens de preferência em número e porcentagem para as 5 montagens e teste qui-quadrado.

\begin{tabular}{c|c|c|c|c|c|c}
\hline \hline \multirow{2}{*}{ Montagem } & \multirow{2}{*}{ Freq. } & \multicolumn{5}{|c}{ Ordem de preferência } \\
\cline { 3 - 7 } & & $1^{\mathrm{o}}$ & $2^{\mathrm{o}}$ & $3^{\mathrm{o}}$ & $4^{\mathrm{o}}$ & $5^{\mathrm{o}}$ \\
\hline \multirow{2}{*}{ Clássica } & $\mathrm{N}^{\mathrm{o}}$ & 27 & 17 & 17 & 4 & 5 \\
\cline { 2 - 7 } & $\%$ & 38,57 & 24,29 & 24,29 & 5,71 & 7,14 \\
\hline \multirow{2}{*}{ Senil } & $\mathrm{N}^{\mathrm{o}}$ & 23 & 17 & 11 & 15 & 4 \\
\cline { 2 - 7 } & $\%$ & 32,85 & 24,28 & 15,72 & 21,43 & 5,72 \\
\hline \multirow{2}{*}{ Masculina } & $\mathrm{N}^{\mathrm{o}}$ & 10 & 8 & 11 & 19 & 22 \\
\cline { 2 - 7 } & $\%$ & 14,28 & 11,42 & 15,72 & 27,15 & 31,43 \\
\hline \multirow{3}{*}{ Diastema } & $\mathrm{N}^{\mathrm{o}}$ & 9 & 23 & 12 & 10 & 16 \\
\cline { 2 - 7 } & $\%$ & 12,85 & 32,85 & 17,15 & 14,29 & 22,86 \\
\hline \multirow{2}{*}{ Feminina } & $\mathrm{N}^{\mathrm{o}}$ & 1 & 5 & 19 & 22 & 23 \\
\cline { 2 - 7 } & $\%$ & 1,42 & 7,14 & 27,15 & 31,43 & 32,86 \\
\hline \hline
\end{tabular}

$\mathrm{x}^{2}=90,57$ (significantemente com nivel de $1 \%$ ). dos votos. Os resultados obtidos por FRIGERIO ${ }^{6}$ (1984) estão de acordo quanto a preferência pela montagem do tipo clássico com $37,60 \%$, mas diferem quanto ao sexo pois encontrou num grupo feminino a montagem do tipo feminino, que obteve $34 \%$ de aprovação, enquanto no nosso grupo masculino, a montagem do tipo masculina obteve $14,28 \%$.

Considerando-se que o experimento foi realizado em pacientes com idade média de 59,1 anos, os resultados estão de acordo com as observações dos autores FENN et al. ${ }^{4}$ (1961), FRUSH; FIS$\operatorname{CHER}^{7,10}$ (1955) e (1957), TAMAKI ${ }^{19}$ (1988), GOLDSTEIN $^{11}$ (1980) que verificaram que a montagem classificada como senil, nesta pesquisa, era a que

TABELA 3 - Testes qui-quadrado para opção de preferência com exclusão sucessiva dos menores valores. Freqüência em porcentagem e números.

\begin{tabular}{|c|c|c|c|c|c|c|}
\hline Opção & Clássica & Senil & $\begin{array}{c}\text { Mascu- } \\
\text { lina }\end{array}$ & $\begin{array}{l}\text { Dias- } \\
\text { tema }\end{array}$ & $\begin{array}{c}\text { Femi- } \\
\text { nina }\end{array}$ & $\begin{array}{c}\text { Qui- } \\
\text { quadrado }\end{array}$ \\
\hline \multirow{5}{*}{$\begin{array}{c}1^{\circ} \\
\text { Lugar }\end{array}$} & 27 & 23 & 10 & 9 & 1 & $32,86^{* *}$ \\
\hline & $38,57 \%$ & $32,85 \%$ & $14,28 \%$ & $12,85 \%$ & $1,42 \%$ & - \\
\hline & 27 & 23 & 10 & 9 & - & $14,42 * *$ \\
\hline & 27 & 23 & 10 & - & - & $7,90 *$ \\
\hline & 27 & 23 & - & - & - & 0,18 n.s. \\
\hline \multirow{5}{*}{$\begin{array}{c}2^{\circ} \\
\text { Lugar }\end{array}$} & 17 & 17 & 8 & 23 & 5 & $5,43 * *$ \\
\hline & $24,29 \%$ & $24,28 \%$ & $11,42 \%$ & $32,85 \%$ & $7,14 \%$ & \\
\hline & 17 & 17 & 8 & 23 & - & 7,06 n.s. \\
\hline & - & - & - & - & - & - \\
\hline & - & - & - & - & - & - \\
\hline \multirow{5}{*}{$\begin{array}{c}3^{\circ} \\
\text { Lugar }\end{array}$} & 17 & 11 & 11 & 12 & 19 & 40,60 n.s. \\
\hline & $24,29 \%$ & $15,72 \%$ & $15,72 \%$ & $17,15 \%$ & $27,15 \%$ & - \\
\hline & - & - & - & - & - & - \\
\hline & - & - & - & - & - & - \\
\hline & - & - & - & - & - & - \\
\hline \multirow{5}{*}{$\begin{array}{c}4^{\circ} \\
\text { Lugar }\end{array}$} & 4 & 15 & 19 & 10 & 22 & $14,71^{* *}$ \\
\hline & $5,71 \%$ & $21,43 \%$ & $27,15 \%$ & $14,29 \%$ & $31,43 \%$ & - \\
\hline & - & 15 & 19 & 10 & 22 & 4,91 n.s. \\
\hline & - & - & - & - & - & - \\
\hline & - & - & - & - & - & - \\
\hline \multirow{5}{*}{$\begin{array}{c}5^{\circ} \\
\text { Lugar }\end{array}$} & 5 & 4 & 22 & 16 & 23 & $23,57 * *$ \\
\hline & $7,14 \%$ & $5,72 \%$ & $31,43 \%$ & $22,86 \%$ & $32,86 \%$ & - \\
\hline & 5 & - & 22 & 16 & 23 & $12,42 * *$ \\
\hline & - & - & 22 & 16 & 23 & 1,41 n.s. \\
\hline & - & - & - & - & - & - \\
\hline
\end{tabular}

n.s. - não significante; *significante ao nivel de 5\%; ${ }^{* *}$ significante ao nivel de $1 \%$. 
CASTRO Jr., O. V. de; HVANOV, Z. V.; FRIGERIO, M. L. M. A. Avaliação estética da montagem dos seis dentes superiores anteriores em prótese total. Pesq Odont Bras, v. 14, n. 2, p. 177-182, abr./jun. 2000.

mais se harmonizava com os indivíduos daquela faixa etária, mas diferiu de FRUSH; FISCHER ${ }^{10}$ (1957), TAMAKI ${ }^{19}$ (1988), HEARTWELL Jr.; RAHN ${ }^{13}$ (1990) quando indicaram diastemas para pacientes idosos que, na presente pesquisa, obtiveram apenas $12,85 \%$, porcentagem essa muito próxima à dos $11,28 \%$ de diastemas encontrados em trabalho de TAMAKI ${ }^{17}$ (1968) com pacientes dentados.

Por outro lado, considerando que todos os pacientes eram do sexo masculino, o resultado difere de autores que acreditam que com os incisivos centrais superiores mais expostos, projetando-se as distais para vestibular e os incisivos laterais superiores mais deslocados levemente para palatino tornando-os menos evidentes, conferiam a montagem mais agressiva e masculina, harmonizando-se com o sexo masculino ${ }^{4,8,18}$.

Examinando-se os resultados apresentados na Tabela 3, as montagens clássicas e com toque de senilidade foram as preferidas em $1^{\circ}$ lugar, com freqüências diferentes $(38,57 \%$ e $32,85 \%$, respectivamente), mas, sem apresentar diferenças estatísticas significantes entre elas.
As montagens que menos agradaram os avaliadores foram as com toque feminino com $32,86 \%$ de desaprovação ( $5^{\circ}$ lugar), embora não tenha ocorrido diferenças estatísticas significantes entre as montagens com toque feminino, com toque masculino e com diastemas.

\section{CONCLUSÕES}

Pela análise dos resultados obtidos pode-se concluir:

1. As montagens que mais agradaram em $1^{\circ}$ lugar foram a do tipo clássico $(38,57 \%)$ e a com toque de senilidade $(32,85 \%)$.

2. Não houve diferença significante entre a montagem do tipo clássica e a com toque de senilidade.

3. A montagem que menos agradou foi a com toque feminino com $32,86 \%$ de desaprovação seguida da montagem masculina $(31,43 \%)$ e com diastema $(22,86 \%)$.

4. Não houve diferença significante entre as montagens com toque feminino, com toque masculino e com diastema.

CASTRO Jr., O. V. de; HVANOV, Z. V.; FRIGERIO, M. L. M. A. Esthetic evaluation of the setting up of the six anterior superior teeth in complete dentures. Pesq Odont Bras, v. 14, n. 2, p. 177-182, abr./jun. 2000.

The purpose of this research was to evaluate different ways of setting up teeth in complete dentures, during the try-in phase (before having them processed). Since this issue has a subjective appeal, the prostheses were submitted to the evaluation of different kinds of people. The different groups of evaluators were: students, specialists and laymen.

UNITERMS: Esthetics, dental; Denture, complete.

\section{REFERÊNCIAS BIBLIOGRÁFICAS}

1. ALDROVANDI, C. Dentaduras completas. 2. ed. Rio de Janeiro : Científica, 1956. v. 2, Cap. 6, p. 190-205.

2. CURTIS, T. A; SHAW, E. L.; CURTIS, D. A. The influence of removable prosthodontic procedures and concepts on the esthetics of complete dentures. J Prosthet Dent, v. 57, n. 1-3, p. 315-323, Mar. 1987.

3. ENGELMEIER, R. L. Complete-denture esthetics. Clin North Am, v. 40, n. 1, p. 71-84, Jan. 1996.

4. FENN, H. R. B; LIDDELOW, K. P.;GRIMSON, A. P. Clinical dental prosthetics. 2. ed. Londres : Staples Press, 1961. p. 417-453.

5. FREITAS, A. G.; SILVA, N. F.; GEOFFROY FILHO, V. M. Prótese total (fases de sua elaboração) III. Rev Bras Odont, v. 31, n. 188, p. 155-159, jul./ago. 1974.

6. FRIGÉRIO, M. L. M. A. Caracterização da montagem dos dentes em prótese total. São Paulo, 1984. p. 11-56 Tese Mestrado em Prótese Dentária pela Faculdade de Odontologia da Universidade de São Paulo.

7. FRUSH, J. P.; FISHER, R. D. Introduction to dentogenic restorations. J Prosthed Dent, v. 5, n. 5, p. 586-595, Sept. 1955.
8. FRUSH, J. P.; FISHER, R. D. How dentogenic restorations interpret the sex factor. J Prosthed Dent, v. 6, n. 2, p. 160-172, July 1956.

9. FRUSH, J. P.; FISHER, R. D. How dentogenic interprets the personality factor. J Prosthed Dent, v. 6, n. 4, p. 441-449, July 1956.

10. FRUSH, J. P.; FISHER, R. D. The age factor in dentogenics. J Prosthed Dent, v. 7, n. 1, p. 5-13, Jan. 1957.

11. GOLDSTEIN, R. E. Estética em Odontologia. Rio de Janeiro : Guanabara Koogan, 1980. p. 2-15.

12. GOMEZ, T.; MORI, M.; CORREAA, G. A. Atlas de caracterização em prótese total e prótese parcial removivel. São Paulo: Santos, 1998. Cap. 3, p. 21.

13. HEARTWELL Jr., C. M.; RAHN, A. O. Syllabus em Dentaduras Completas. Trad. de José Cerrati Turano e Fernando Montenegro. 4. ed. São Paulo : Santos, 1990. Cap. 14 , p. 343-353.

14. NICACIO, I. M. Considerações sobre a seleção dos dentes artificiais em prótese total. Rev Ass Paul Cirurg Dent, v. 30, p. 358-62, nov./dez. 1976.

15. RUFENACHT, C. R. Fundamentals of esthetics. Berlin : Quintessenz, 1990. Cap. 5, p. 137-183. 
CASTRO Jr., O. V. de; HVANOV, Z. V.; FRIGERIO, M. L. M. A. Avaliação estética da montagem dos seis dentes superiores anteriores em prótese total. Pesq Odont Bras, v. 14, n. 2, p. 177-182, abr./jun. 2000.

16. SAITO, T.; CORREAA, G. A.; MORI, M.; YOSHIDA, H.; MORAES, S. L. D.; TAMAKI, R.; GOMEZ, T. Caracterização de dentes de estoque para prótese total. Rev Bras Odontol, v. 1, n. 1, p. 46-49, jul./set. 1993.

17. TAMAKI, S. T. Fator estético em dentaduras completas. Estomat Cult, v. 2, n. 1, p. 39-45, jan./jun. 1968.

18. TAMAKI, T.; TAMAKI, S. T. Prática de laboratório: den- taduras completas. 2. ed. São Paulo: Sarvier, 1980. $91 \mathrm{p}$.

19. TAMAKI, T. Dentaduras completas. 4. ed. rev. ampl. São Paulo : Savier, 1988. p. 194-195.

20. TODESCAN, R. Estética e caracterização de dentes e gengivas na dentadura. Rev Assoc Paul Cir Dent, v. 10, n. 3, p. 158-162, maio/jun. 1956.

Recebido para publicação em 08/09/99

Enviado para reformulação em 09/11/99 Aceito para publicação em 17/01/00 
CASTRO Jr., O. V. de; HVANOV, Z. V.; FRIGERIO, M. L. M. A. Avaliação estética da montagem dos seis dentes superiores anteriores em prótese total. Pesq Odont Bras, v. 14, n. 2, p. 177-182, abr./jun. 2000.

TABELA 1 - Frequência em números dos resultados dos votos quanto a preferência das 5 montagens (pacientes do sexo masculino).

\begin{tabular}{|c|c|c|c|c|c|c|c|c|}
\hline \multirow{2}{*}{$\begin{array}{l}\text { Or- } \\
\text { dem }\end{array}$} & \multirow{2}{*}{ Pac. } & \multirow{2}{*}{$\begin{array}{l}\text { Idade } \\
\text { (anos) }\end{array}$} & \multirow{2}{*}{ Montagem } & \multicolumn{5}{|c|}{ Ordem de Preferência } \\
\hline & & & & $1^{\circ}$ & $2^{\circ}$ & $3^{\circ}$ & $4^{\circ}$ & $5^{\circ}$ \\
\hline \multirow{5}{*}{1} & \multirow{5}{*}{ H.N. } & \multirow{5}{*}{62} & Diastema & 0 & 3 & 0 & 0 & 4 \\
\hline & & & Clássica & 4 & 0 & 2 & 1 & 0 \\
\hline & & & Senil & 3 & 0 & 1 & 3 & 0 \\
\hline & & & Feminina & 0 & 1 & 4 & 2 & 0 \\
\hline & & & Masculino & 0 & 3 & 0 & 1 & 3 \\
\hline \multirow{5}{*}{2} & \multirow{5}{*}{ P.S. } & \multirow{5}{*}{72} & Diastema & 0 & 3 & 3 & 1 & 0 \\
\hline & & & Clássica & 2 & 2 & 1 & 1 & 1 \\
\hline & & & Senil & 4 & 2 & 0 & 1 & 0 \\
\hline & & & Feminina & 0 & 0 & 1 & 3 & 3 \\
\hline & & & Masculino & 1 & 0 & 2 & 1 & 3 \\
\hline \multirow{5}{*}{3} & \multirow{5}{*}{ P.P. } & \multirow{5}{*}{62} & Diastema & 0 & 3 & 1 & 1 & 3 \\
\hline & & & Clássica & 5 & 1 & 1 & 0 & 1 \\
\hline & & & Senil & 2 & 1 & 2 & 2 & 1 \\
\hline & & & Feminina & 0 & 1 & 2 & 2 & 3 \\
\hline & & & Masculino & 1 & 2 & 2 & 3 & 0 \\
\hline \multirow{5}{*}{4} & \multirow{5}{*}{ E.P.S. } & \multirow{5}{*}{18} & Diastema & 3 & 2 & 1 & 1 & 0 \\
\hline & & & Clássica & 0 & 3 & 1 & 0 & 3 \\
\hline & & & Senil & 1 & 1 & 1 & 3 & 1 \\
\hline & & & Feminina & 0 & 0 & 3 & 2 & 2 \\
\hline & & & Masculino & 3 & 1 & 1 & 1 & 1 \\
\hline \multirow{5}{*}{5} & \multirow{5}{*}{ M.F.A } & \multirow{5}{*}{51} & Diastema & 0 & 2 & 2 & 0 & 2 \\
\hline & & & Clássica & 0 & 3 & 3 & 0 & 0 \\
\hline & & & Senil & 6 & 0 & 0 & 0 & 0 \\
\hline & & & Feminina & 0 & 1 & 1 & 3 & 1 \\
\hline & & & Masculino & 0 & 0 & 0 & 3 & 3 \\
\hline
\end{tabular}

TABELA 1 (cont.) - Frequência em números dos resultados dos votos quanto a preferência das 5 montagens (pacientes do sexo masculino).

\begin{tabular}{|c|c|c|c|c|c|c|c|c|}
\hline \multirow{2}{*}{$\begin{array}{l}\text { Or- } \\
\text { dem }\end{array}$} & \multirow{2}{*}{ Pac. } & \multirow{2}{*}{$\begin{array}{l}\text { Idade } \\
\text { (anos) }\end{array}$} & \multirow{2}{*}{ Montagem } & \multicolumn{5}{|c|}{ Ordem de Preferência } \\
\hline & & & & $1^{\circ}$ & $2^{\circ}$ & $3^{\circ}$ & $4^{\circ}$ & $5^{\circ}$ \\
\hline \multirow{5}{*}{6} & \multirow{5}{*}{ R.B. } & \multirow{5}{*}{66} & Diastema & 2 & 2 & 0 & 3 & 0 \\
\hline & & & Clássica & 3 & 0 & 3 & 1 & 0 \\
\hline & & & Senil & 1 & 3 & 1 & 0 & 2 \\
\hline & & & Feminina & 0 & 2 & 0 & 1 & 4 \\
\hline & & & Masculino & 1 & 0 & 3 & 2 & 1 \\
\hline \multirow{5}{*}{7} & \multirow{5}{*}{ R.G.L. } & \multirow{5}{*}{62} & Diastema & 0 & 0 & 3 & 2 & 2 \\
\hline & & & Clássica & 6 & 1 & 0 & 0 & 0 \\
\hline & & & Senil & 1 & 5 & 0 & 1 & 0 \\
\hline & & & Feminina & 0 & 0 & 2 & 1 & 4 \\
\hline & & & Masculino & 0 & 1 & 2 & 3 & 1 \\
\hline \multirow{5}{*}{8} & \multirow{5}{*}{ D.S. } & \multirow{5}{*}{70} & Diastema & 0 & 2 & 0 & 1 & 3 \\
\hline & & & Clássica & 1 & 1 & 4 & 0 & 0 \\
\hline & & & Senil & 2 & 2 & 1 & 1 & 0 \\
\hline & & & Feminina & 0 & 0 & 0 & 3 & 3 \\
\hline & & & Masculino & 3 & 1 & 1 & 1 & 0 \\
\hline \multirow{5}{*}{9} & \multirow{5}{*}{ W.A. } & \multirow{5}{*}{60} & Diastema & 0 & 4 & 1 & 1 & 2 \\
\hline & & & Clássica & 6 & 1 & 1 & 0 & 0 \\
\hline & & & Senil & 1 & 3 & 3 & 1 & 0 \\
\hline & & & Feminina & 0 & 0 & 3 & 3 & 2 \\
\hline & & & Masculino & 1 & 0 & 0 & 3 & 4 \\
\hline \multirow{5}{*}{10} & \multirow{5}{*}{ J.R.F. } & \multirow{5}{*}{68} & Diastema & 4 & 2 & 1 & 0 & 0 \\
\hline & & & Clássica & 0 & 5 & 1 & 1 & 0 \\
\hline & & & \begin{tabular}{|l|} 
Senil \\
\end{tabular} & 2 & 0 & 2 & 3 & 0 \\
\hline & & & Feminina & 1 & 0 & 3 & 2 & 1 \\
\hline & & & Masculino & 0 & 0 & 0 & 1 & 6 \\
\hline
\end{tabular}

